\title{
BLOCKCHAIN PARA OPERAÇÕES INTERBANCÁRIAS
}

\author{
Raphael Alves Bruce, Rafael Timóteo de Sousa Júnior, Fábio Lúcio Lopes de Mendonça, \\ João Paulo Pimentel, Maristela Terto de Holanda e Francisco Lopes de Caldas Filho \\ Programa de Pós-Graduação em Engenharia Elétrica (PPGEE) - Universidade de Brasília, Brasília, Brasil
}

\begin{abstract}
RESUMO
O blockchain surgiu como a principal tecnologia utilizada pelas criptomoedas e rapidamente teve seu uso considerado em várias outras soluções devido a transparência, disponibilidade, segurança e descentralização. Tais características motivam seu uso como solução para operações interbancárias em substituição às interface legadas baseadas no processamento em lote de transações enviadas por arquivos. Esse artigo apresenta um modelo de blockchain de consórcio que adapta mecanismos do blockchain do Bitcoin superando seus problemas de escalabilidade, segurança, throughput e privacidade. No modelo proposto, segurança e privacidade são garantidas pelo modelo federado e por soluções da camada de autenticação, escalabilidade é feita por mecanismo de consenso customizado menos oneroso, e desempenho é alcançado pelo design do processo de transações otimizado para o contexto de uso.
\end{abstract}

\section{PALAVRAS-CHAVE}

Blockchain de Consórcio, Operações Interbancárias

\section{INTRODUÇÃO}

Várias entidades financeiras realizam diariamente operações entre si e que são comunicadas a uma autoridade central, a qual garante o funcionamento do sistema como um todo. A maioria dessas transações são comandadas de forma online, mas sua efetivação só acontece várias horas depois, após o processamento em lote das transações de determinado produto, o que geralmente ocorre fora da janela habitual de atendimento comercial.

O mecanismo de interface mais comum entre instituições bancárias envolve os arquivos sequenciais. Assim, as transações de determinado produto vão sendo realizadas durante uma determinada janela de horário comercial, para serem agrupadas em um arquivo de interface a ser transmitido para outra instituição, que fará o processamento de cada transação aplicando as regras de negócio relacionadas e enviará um outro arquivo de retorno com o resultado desse processamento à instituição de origem.

As operações interbancárias funcionam em sua maioria utilizando soluções legadas em que cada nó participante da operação negocial possui sua base de dados, sendo a transparência da operação muito baixa visto que as regras de processamento são mantidas de forma centralizada por cada unidade processadora. Dessa maneira, os mecanismos atuais de operações interbancárias são entraves para auditoria constante, verificação de conformidade permanente, efetivação imediata do serviço contratado, transparência das ações de contratação e economia dos valores gastos com as verificações pela autoridade central.

\section{REVISÃO SOBRE BLOCKCHAIN}

O cenário de interface de sistemas entre empresas, entidades financeiras e órgãos reguladores para execução de um fluxo de negócio compartilhado de determinado produto é bastante comum, sendo urgente a interoperabilidade por meio de soluções altamente disponíveis, garantindo imutabilidade dos dados compartilhados, escalabilidade e desempenho, privacidade dos dados pessoais, auditoria e rastreabilidade (L. Adrian et al, 2018).

No blockchain o principal modelo de referência é o Bitcoin. Se trata de uma criptomoeda cujo o blockchain funciona como um banco de dados distribuído (sem autoridade central), no qual os registros são mantidos em blocos vinculados como uma cadeia, conhecida como livro-razão, em que cada bloco contém, pelo menos, o 
hash criptográfico do cabeçalho do bloco anterior, o timestamp atual e o hash das transições registradas, (N. Satoshi, 2008).

O blockchain do Bitcoin é público, pois pode ser acessado por qualquer pessoa que baixe seu livro-razão. Foi estabelecido com a intenção de obter confiança nas interações dos participantes de uma rede distribuída pelo consenso da maioria dos nós e pela transparência do processo. Assim, as cópias ficam distribuídas nos participantes da rede e a persistência dos blocos da cadeia ocorre após um processo de verificação oneroso utilizando o mecanismo de prova de trabalho (N. Satoshi, 2008). O blockchain do Bitcoin possui as seguintes características: transparência, consenso, disponibilidade, segurança e descentralização. Seus respectivos detalhes podem ser observados em (K. Akanksha et al, 2018).

\subsection{Discussão sobre Blockchain para Operações Interbancárias}

A discussão visa fundamentar alguns pontos de utilização do blockchain de forma a se ter uma solução para as operações interbancárias entre entidades financeiras com alta disponibilidade, baixa latência, auditoria constante e sem a necessidade de validação por órgão terceiro.

O blockchain do Bitcoin apresenta qualidades contundentes como solução tecnológica, porém, ainda assim, os mesmos mecanismos que proporcionam as qualidades anteriormente identificadas geram desvantagens, entre as quais: falta de escalabilidade, consumo excessivo de tempo, alto custo computacional, excessiva replicação de dados, alta latência, questões com tamanho e largura de banda. A falta de escalabilidade e a excessiva replicação advêm da necessidade de manter a cadeia em cada nó para participar da rede, pois a capacidade de armazenamento e poder computacional exigidos dos membros da rede acaba sendo muito grande conforme seu crescimento (Z. Zibin, 2018).

O consumo excessivo de tempo e processamento advém do mecanismo de prova de trabalho, que consome bastante tempo e poder de processamento para resolução dos cálculos criptográficos envolvidos no processo de hashing. Já a alta latência ocorre pela natureza da cadeia que exige serialização na inserção de registros e pela complexidade no mecanismo de consenso. E as questões com relação ao tamanho e largura de banda estão relacionadas às definições arbitrárias da solução quanto ao tamanho do bloco de até $1 \mathrm{Mb}$ e a latência de um bloco a cada 10 minutos, limitando o número de transações à média de 500 por bloco (I. Eyal et al, 2016).

O blockchain é uma alternativa às soluções tradicionais de operações interbancárias, para que as instituições possam compartilhar bancos de dados distribuídos, ou seja, o livro-razão de determinados produtos. Em um contexto que não há confiança entre as partes, mas que as transações são verificadas pela solução compartilhada de forma transparente, sem a necessidade de validação por uma autoridade reguladora, a qual, caso exista, pode ser apenas comunicada sobre as operações ou acessar ao blockchain para auditoria e monitoramento.

Assim, diminui-se os pontos de possibilidade de falha nas operações interbancárias e gera-se economia pela redução do desenvolvimento de soluções para processar as transações em cada nó das interfaces e pela possível dispensa ou renegociação dos termos de atuação da entidade verificadora.

\section{DESCRIÇÃO BÁSICA DO MODELO}

No modelo proposto, a primeira questão a ser considerada é a governança geral da blockchain, que pode ser pública, privada ou híbrida (também é conhecida como de consórcio ou de comunidade), considerando a característica de permissão que os nós participantes da rede possuem (T. Paolo, 2017).

Uma solução para realizar operações interbancárias deve utilizar a blockchain de consórcio, em que os nós participantes serão as instituições que entrarem em acordo para compartilhar a rede. Neste caso, apenas as corporações que façam parte do consórcio da blockchain terão permissão de gravação na cadeia. É um contexto em que há um certo nível de confiança devido ao acordo jurídico para atingir o interesse comum de compartilhar um fluxo negocial visando lucro, porém a confiança não é total.

Como a blockchain de consórcio não é uma rede totalmente pública, não há necessidade dos processos de mineração, prova de trabalho e recompensa (G. Dominique, 2017). A blockchain de consórcio ainda permite a definição de tamanhos de blocos e cadeias, participantes da determinação do consenso, graus de permissão e procedimentos de paralelização. Como os participantes da rede estão em consórcio, soluções onerosas de hash podem ser substituídas por camadas e controles de acesso que se adaptam às necessidades das empresas, aumentando a segurança, a escalabilidade e a privacidade. Ainda prevê-se o uso de smart contracts (programas 
imutáveis implantados e executados de forma descentralizada no blockchain, para a implementação de diretrizes que atendam às necessidades do consórcio).

Neste modelo de consórcio deve haver ainda uma entidade auditora, que não seja controlada por nenhuma das empresas participantes do acordo, mas constituída ou outorgada por elas. O papel de auditoria pode ser realizado pela implementação de smart contracts que executem as regras de conformidade e monitoramento. Esta realização é importante para evitar que participantes legítimos do consórcio executem ou promovam acordos paralelos para a realização de ações fraudulentas.

O modelo de blockchain de consórcio ainda possui a vantagem de ter um número reduzido de participantes em comparação ao blockchain público e não depender de uma autoridade central como o blockchain privado, sendo a responsabilidade de verificação das transações dividida entre participantes que se conhecem e estão em acordo. Assim, esta abordagem apresenta as seguintes vantagens: a) mais velocidade nas transações, pois os procedimentos de verificação podem ser realizados em paralelo por um conjunto autorizado de nós; b) maior escalabilidade pela paralelização e ausência de disputa entre os nós para participar; e c) menos gasto de tempo e poder computacional, já que não é responsabilidade do procedimento de verificação se preocupar com ataques de segurança, uma vez que os nós participantes foram validados e autorizados. Esta responsabilidade pode ser garantida pela entidade auditora, mantendo smart contracts para garantir que apenas entidades autorizadas, ou seja, participante do consórcio, insira registros na cadeia.

Neste modelo, o mecanismo de verificação das transações não depende da prova de trabalho e pode ser realizada por protocolo do tipo Practical Byzantine Fault Tolerance (PBFT), que é um algoritmo de Byzantine Fault Tolerance (BFT) que alcança consenso em três rodadas de troca de mensagens entre os nós, garantindo que $3 \mathrm{~N}+1$ dos nós possam obter consenso também na presença de $\mathrm{N}$ nós bizantinos. Entende-se que algoritmo BFT conhecido como prova de autoridade é mais indicado para blockchain de consórcio, pois requer menos troca de mensagens. Os algoritmos de prova de autoridade contam com um conjunto de $\mathrm{N}$ autoridades certificadas, sendo que uma delas é eleita líder de determinada rodada de consenso, para transmitir um ciclo de mensagens para obter consenso por N/2+1. Assim, a disponibilidade é aprimorada e a consistência é eventual, nos casos de indisponibilidade de um número maior que o limite para atender contextos bizantinos ou da recorrência de indisponibilidade do líder da rodada, mas isso pode ser aprimorado com a implementação de smart contracts, evitando possíveis ramificações na rede após sua ocorrência. Finalmente, usando um protocolo BFT é alcançado o consenso determinístico, para que as transações sejam imediatamente confirmadas ou rejeitadas a partir do blockchain (A. Stefano, 2018).

Outra possibilidade interessante é a de substituir a Merkle Tree do Bitcoin (N. Satoshi, 2008) como estrutura de dado do cabeçalho do bloco pelo Practical Algorithm To Retrieve Information Coded In Alphanumeric (Patricia) Merkle Tree, que tem a vantagem de ser mais flexível ao permitir a verificação de ramos específicos da árvore, invés de verificação de um ramo de cada nível como o Merkle Tree. Isso dá liberdade de estabelecer processos mais ou menos rígidos conforme a quantidade de ramos selecionados no processo de verificação, de acordo com a relação de confiança entre os membros do consórcio, quanto maior a desconfiança, mais rígida a implementação de verificação do Patricia Merkle Tree.

Outra implementação que ajuda na escalabilidade e no alto desempenho da resposta é tornar os limites de tamanho e largura de banda dos blocos customizados. Essa abordagem pode ser usada ainda para definir o limite por número de usuários e o limite por número de nós participantes, tornando o consenso mais fácil ou difícil de alcançar, dependendo da regra de negócios atendida.

Existem várias pesquisas que exploram problemas com ataques de segurança (K. Akanksha et al, 2017). No entanto, esses ataques iminentemente não afetam o blockchain de consórcio, uma vez que não é aberto a qualquer participante, mas ainda assim deve haver uma preocupação com os nós perversos. Logo, para comunicação, a blockchain de consórcio deve confiar em seu sistema Public Key Infrastructure (PKI), tendo cuidado com os ataques de alguns adversários, como por exemplo, comprometimento da autoridade certificadora sem detecção, emissão de certificado não autorizado para domínio malicioso e status de revogação de certificado não autêntico.

A blockchain de consórcio proposta adota um modelo federado para credenciamento dos participantes, que devem ser autenticados em uma camada de segurança usando o padrão OAuth 2.0 (D. Hardt, 2012). Esse processo complementará o conceito de sindicalização, pelo qual uma autoridade central ou colegiada é capaz de interpretar o modelo federado para extrair informações significativas e implementar diretivas de regulamentação para executar a proteção da rede. $\mathrm{O}$ modelo federado é recomendado para blockchain de consórcio, pois é um padrão de arquitetura corporativa, permitindo a interoperabilidade e o compartilhamento de informações entre linhas de negócios e sistemas de tecnologia da informação. 
O modelo proposto deve adotar em sua camada de segurança uma proteção contra smart contracts com intenção fraudulenta por duas diretrizes complementares: a) adotar um controle intensivo e um monitoramento exaustivo com relação à implantação de smart contracts no blockchain, tornando este processo o mais transparente possível; e b) manter smart contracts de auditoria, buscando possíveis ações fraudulentas efetuadas pelos participantes mal-intencionados, considerando os parâmetros definidos pelo consórcio. O monitoramento da rede é fundamental e as diretrizes de segurança devem ser adaptáveis considerando as ações executadas pelos nós para que um protocolo seja aplicado em circunstâncias normais e um mais rígido seja ativado para os nós que estão agindo longe de seus parâmetros normais.

Dada a governança mais centralizada da blockchain de consórcio e sua natureza menos exposta, a privacidade não é problema urgente, mesmo assim, é recomendado na implementação do modelo proposto um mecanismo que estabeleça o grau de anonimato para cada registro da blockchain, especificando quem pode acessar o quê, o que significa, que as informações só estarão disponíveis após a aprovação pelos nós competentes e a validação das credenciais, em um modelo federado que possui autenticação descentralizada fornecida pela autoridade de certificação do consórcio.

\section{CONCLUSÃO}

O modelo proposto busca melhorar escalabilidade, segurança, throughput e privacidade, e apresenta várias vantagens sobre o processo legado das operações interbancária, garantindo transparência, autonomia no monitoramento e verificação, disponibilidade, rastreabilidade e colaboração. Vantagens que refletem em redução de custos pelo compartilhamento da solução e a dispensa da verificação por terceiros, além da diminuição do trabalho de backoffice exigido pelas soluções legadas, que exigem vários processos de conformidade no acompanhamento das operações interbancárias e seus reflexos.

Pontos que devem ser observados em trabalhos futuros são as definições dos limites dos participantes do processo de validação das transações para ser o mais eficiente possível e a utilização dos smart contracts como auditores de blockchain de consórcios.

\section{AGRADECIMENTO}

Os autores agradecem o apoio das Agências brasileiras de pesquisa, desenvolvimento e inovação CNPq (Projeto INCT em Segurança Cibernética 465741/2014-2), CAPES (Projeto FORTE 23038.007604/2014-69 e PROBRAL 88887.144009/2017-00) e Fundação de Apoio à Pesquisa do Distrito Federal FAPDF (Projetos UIoT 0193.001366/2016 e SSDDC 0193.001365/2016) e do CCA-BR (Centro de Computação da Aeronáutica de Brasília) juntamente ao ComDeCiber (Comando de Defesa Cibernética), bem como do Gabinete de Segurança Institucional da Presidência da República (TED 002/2017) e do Laboratório LATITUDE/UnB (Projeto SDN 23106.099441/2016-43).

\section{REFERÊNCIAS}

Adrian, L. et al, 2018. Predicts 2019: Blockchain Business. December's Gartner Release.

Akanksha, K. et al, 2017, Blockchain - Literature Survey. 2nd IEEE International Conference On Recent Trends in Electronics Information \& Communication Technology.

Dominique, G., 2017, Public Blockchain versus Private Blockchain. Journal Centre d'économie de la Sorbonne May.

Eyal, I. et al, 2016, Bitcoin-NG: A Scalable Blockchain Protocol. 13th USENIX Symposium on Networked Systems Design and Implementation.

Hardt, D., 2012. The OAuth 2.0 Authorization Framework. RFC 6749.

Paolo, T. et al, 2017, Ontology of Blockchain Technologies: Principles of Identification and Classification. SSRN Electronic Journal.

Satoshi, N., 2008, A Peer-to-peer Eletronic Cash System. Disponível em: https://bitcoin.org/files/bitcoinpaper/bitcoin_pt_br.pdf.

Stefano, A., 2018, PBTF vs Proof-of-Authority: applying the CAP theorem to permissioned blockchain. Italian Conference on Cyber Security.

Zibin, Z., 2018, Blockchain Challenges and Opportunities: A Survey. International Journal of Web and Grid Services. 